\title{
Engineering and expression of a human rotavirus candidate vaccine in Nicotiana benthamiana
}

Francisco F. P. G. Pêra', David L. R. Mutepfa ${ }^{1}$, Ayesha M. Khan ${ }^{1}$, Johann H. Els ${ }^{1}$, Sandiswa Mbewana ${ }^{1}$, Alberdina A. A. van Dijk², Edward P. Rybicki ${ }^{1,3}$ and Inga I. Hitzeroth ${ }^{1 *}$

\begin{abstract}
Background: Human rotaviruses are the main cause of severe gastroenteritis in children and are responsible for over 500000 deaths annually. There are two live rotavirus vaccines currently available, one based on human rotavirus serotype G1P[8], and the other a G1-G4 P[8] pentavalent vaccine. However, the recent emergence of the G9 and other novel rotavirus serotypes in Africa and Asia has prompted fears that current vaccines might not be fully effective against these new varieties.

Results: We report an effort to develop an affordable candidate rotavirus vaccine against the new emerging G9P[6] (RVA/Human-wt/ZAF/GR10924/1999/G9P[6]) strain. The vaccine is based on virus-like particles which are both highly immunogenic and safe. The vaccine candidate was produced in Nicotiana benthamiana by transient expression, as plants allow rapid production of antigens at lower costs, without the risk of contamination by animal pathogens. Western blot analysis of plant extracts confirmed the successful expression of two rotavirus capsid proteins, VP2 and VP6. These proteins assembled into VLPs resembling native rotavirus particles when analysed by transmission electron microscopy (TEM). Expression of the rotavirus glycoprotein VP7 and the spike protein VP4 was also tried. However, VP7 expression caused plant wilting during the course of the time trial and expression could never be detected for either protein. We therefore created three fusion proteins adding the antigenic part of VP4 (VP8*) to VP6 in an attempt to produce more appropriately immunogenic particles. Fusion protein expression in tobacco plants was detected by western blot using anti-VP6 and anti-VP4 antibodies, but no regular particles were observed by TEM, even when co-expressed with VP2.
\end{abstract}

Conclusion: Our results suggest that the rotavirus proteins produced in $N$. benthamiana are candidates for a subunit vaccine specifically for the G9P[6] rotavirus strain. This could be more effective in developing countries, thereby possibly providing a higher overall efficacy for the existing vaccines. The production of rotavirus proteins in plants would probably result in lower manufacturing costs, making it more affordable for developing countries. Further investigation is required to evaluate the immunogenic potential of the VLPS and fusion proteins created in this study.

\footnotetext{
* Correspondence: inga.hitzeroth@uct.ac.za

'Biopharming Research Unit, Department of Molecular and Cell Biology,

University of Cape Town, Rondebosch, Cape Town, South Africa

Full list of author information is available at the end of the article
} 


\section{Background}

Rotavirus (RV) infection has probably been a problem as long as humankind has existed, but the connection between RV as the leading cause of severe diarrhoeal disease and dehydration in children under the age of five worldwide was only made in the 1970s [1]. The disease accounts for one third of hospitalizations for diarrhoea worldwide and results in over 500000 child deaths per year in under 5-year olds, with mortality greatest in south Asia and sub-Saharan Africa [2-6].

Rotaviruses are non-enveloped viruses in the family Reoviridae, genus Rotavirus. Virions are triple-layered and contain a viral genome that consists of 11 segments of double-stranded RNA (dsRNA). These dsRNAs encode six structural proteins (VPs) and six non-structural proteins (NSPs). The capsid is composed of an inner VP2 layer, VP6 forming the intermediate capsid, and an outer layer made of VP7. VP4 forms 60 spikes on the outer surface of the virus (Fig. 1). The VP7 layer is essential for incorporation of VP4 into particles and for the virion to be able to infect a cell. The spike protein VP4 gets cleaved by proteases located in the host intestinal lumen into VP5* and VP8* [7]. VP8* is highly immunogenic and has been proven to elicit high levels of homotypic and heterotypic neutralising antibodies when injected into mice [8]. The neutralizing epitope of VP8* was produced in tobacco plants and has shown to confer protection against bovine rotavirus infection in a mouse model [9].

Rotaviruses are divided into 7 groups (A-G) and four subgroups (I, II, I + II and Non I/II) in group A which are based on the antigenic properties of VP6 [10, 11]. The 2 outer capsid proteins define the dual serotype classification of the viruses, with VP4 (protease-sensitive) defining the P serotype, and VP7 (a glycoprotein) the G

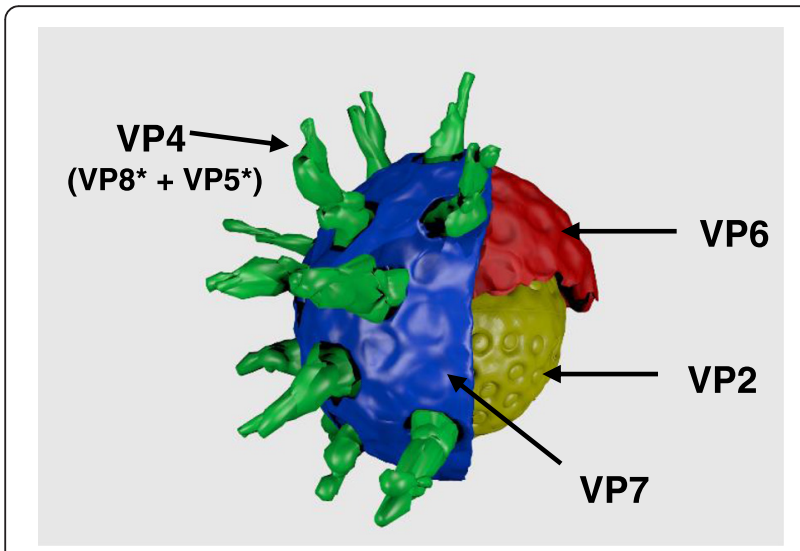

Fig. 1 Schematic representation of a rotavirus virion. The virus is composed of an outer capsid (VP7 and VP4), an intermediate capsid (VP6), and an inner capsid (VP2). The outer capsid protein VP4 gets cleaved by proteases located in the host intestinal lumen into VP5* and VP8*. The average size of a rotavirus particle is $70 \mathrm{~nm}$ serotype [12, 13]. Presently 27 different G-genotypes and 35 P-genotypes have been described, with at least $73 \mathrm{G} / \mathrm{P}$ genotype combinations having been found. More recently a classification system that encompasses all $11 \mathrm{RV}$ gene segments has been introduced, and two major genotype (non-G, non-P) constellations termed Wa-like and DS-1like have been identified $[13,14]$.

Vaccination is the best control measure to protect against disease, but prior to specific rotavirus vaccine development, the most common serotypes occurring in specific geographic locations need to be determined in order to develop a broadly effective vaccine. There are currently two licensed vaccines available on the market: GlaxoSmithKline's Rotarix ${ }^{\odot}$ and Merck's RotaTeq ${ }^{\mathrm{Tm}}$. Both of these vaccines are given orally and consist of of live attenuated rotavirus that replicates inside the small intestine. Rotarix ${ }^{\bullet}$ contains the most commonly occurring human rotavirus strain globally, G1P[8]. RotaTeq ${ }^{\text {Ts }}$ consists of a reassortant collection of 5 viruses comprising 10 bovine rotavirus genes and 5 human rotavirus genes, protecting against the four most prevalent human strains of rotavirus; namely, G1, G2, G3 and G4 and serotype $P[4]$ [15]. A study that reviewed the rotavirus strain types that were found to be circulating in Africa over a 10 year period (1997-2006) revealed that there was an increase in RV strain diversity in Africa, with the recent emergence and spread of the G9 serotype [16-18]. Other studies noted the emergence of G2, G8 and G9 rotaviruses and unusual $\mathrm{G}$ and $\mathrm{P}$ combinations and a high prevalence of a P[6] VP4 genotype $[11,19]$. Emergence of the G9 and novel rotavirus strains in Africa prompted fears that the current vaccines might not protect against these new serotypes, and it is thought that these rotavirus strains need to be taken into account when developing new or better vaccines against rotavirus.

A clinical trial with Rotarix $^{\odot}$ (G1P[8]) in 2006-2007 showed that this vaccine had a $49.9 \%$ efficacy in Malawi and $76.9 \%$ vaccine efficacy in South Africa [20]. The efficacy of RotaTeq ${ }^{\mathrm{Tm}}$, the pentavalent rotavirus vaccine, was also only $64.2 \%$ in the first year of life; overall vaccine efficacy against severe rotavirus gastroenteritis was $39 \%$ in developing countries in sub-Saharan Africa, but higher $(68-75 \%)$ in developed countries [21, 22]. The efficacy against G9 specifically was $49.7 \%$. The low efficacy of the two rotavirus vaccines in Africa compared to more developed countries highlights the need to develop a vaccine that will be region-specific and will include prevalent $G$ and $P$ genotypes in the vaccine $[23,24]$. Accordingly, in this work we endeavoured to express rotavirus proteins from the new emerging G9P[6] (RVA/ Human-wt/ZAF/GR10924/1999/G9P[6]) strain which was recently sequenced and analysed [11, 25].

One disadvantage of live attenuated vaccines remains viral shedding and gene reassortment [26]. An alternative 
is non-infectious vaccines based on virus-like particles (VLPs): these contain only the viral capsid proteins, with no viral genomic material, and mimic the native rotavirus virion in antigenicity. An important criterion for the use of VLPs as vaccine candidates is their safety. Rotavirus VLPs produced in insect cells and yeast have received attention in recent years as alternative vaccine candidates that are highly immunogenic and safe [27-30].

Plants have recently been utilized as an alternative production system which is suited for the rapid production of antigens at lower costs, and without the risk of contamination by animal pathogens [31]. Various groups have expressed rotavirus capsid proteins in plants. Matsumura (2002) reported bovine rotavirus A VP6 expression in transgenic potato plants. The protein was purified and immunogenicity studies performed: adult mice developed anti-VP6 antibodies in their sera. However, there was no evidence of assembly of the VP6 and immune responses may have been to simple monomers or trimers [32]. Other workers showed assembly of VP6 expressed in N. benthamiana via a potato virus X (PVX)-derived vector. The VP6 formed trimers, assembled around VP2 cores, and still assembled when fused to the PVX CP, as protein rods. Once cleaved from PVX CP, the VP6 assembled into icosahedral VLPs [33]. A more recent study showed the successful expression of codon-optimized human rotavirus VP6 in Chenopodium amaranticolor using a Beet black scorch virus (BBSV)-mediated expression system with the VP6 gene replacing the CP gene of BBSV. Oral immunization of female BALB/c mice with the plant based VP6 protein induced high titres of anti-VP6 mucosal IgA and serum IgG [34]. The paper did not mention, however, whether or not the VP6 proteins assembled into VLPs. Saldana et al. (2006) successfully expressed VP2 and VP6 in the cytoplasm of fruits from transgenic tomato plants [35]. Electron microscopy showed that a small proportion of the particles had assembled into $2 / 6$ VLPs. A protective immune response was detected in mice; however, this may have to some extent been contributed by the non-assembled VPs.

The above studies showed that rotavirus coat proteins can be expressed in relatively high levels in plants; that VP2 and VP6 are capable of forming VLPs in plants, and that these VLPs elicit protective immune responses in animal models. In this work, we report an effort to express several rotavirus proteins in plants via transient agroinfiltration-mediated expression in $N$. benthamiana leaves. These proteins could be considered in the future as candidates for an affordable rotavirus VLP vaccine against the new emerging G9P[6] strain. We investigated the effect of intracellular targeting on expression levels of VP6 by targeting the protein to the ER, apoplastic spaces, chloroplast or cytosol. We also fused the highly immunogenic VP8* or the neutralising epitope of VP8* to VP6 and co-expressed these chimeric proteins together with VP2. We further determined the ability of these proteins to form VLPs by electron microscopy.

\section{Results and discussion}

\section{Expression of Rotavirus recombinant proteins in tobacco} plants

All rotavirus proteins used for this study derive from the G9 P[6] strain which is predominant in South Africa and other sub-Saharan regions [21, 22]. A VLP vaccine targeting this strain would help in alleviating the burden of disease in countries from this region.

Agrobacterium tumefaciens cultures containing the recombinant proteins were co-expressed with or without the Tomato spotted wilt virus (TSWV) silencing suppressor protein NSs in N.benthamiana to optimise expression in plant cells. NSs is a RNA silencing suppressor protein which inhibits the onset of post-transcriptional gene silencing (PTGS) [36]. Leaf discs were harvested at different time points to determine the optimum day of expression. In an attempt to boost protein yields, we initially tried directing proteins to different intracellular compartments by using several targeting tags. Infiltrating the plants with Agrobacterium at different optical densities (ODs) was also done to determine the optimum OD for highest protein expression.

The intermediate capsid protein VP6 was successfully expressed using the plant expression vectors from the pTRA family. The cytoplasm-targeted $40 \mathrm{kDa}$ VP6 was present from day one of the time trial, with increasing protein accumulation during the week-long trial (Fig. 2a). Adding NSs only enhanced cytoplasmic accumulation of VP6 at day 7. Protein accumulation of VP6 in the chloroplasts occurred between days 1 and 3 . In contrast to cytoplasmic accumulation, addition of NSs had a considerable effect on accumulation in chloroplasts, as no VP6 was observed in its absence. There was also no detectable protein at days 5 and 7 (Fig. 2c). The apoplast and the ER showed best protein accumulation at day 3 of the time trial and none at all on days 1 and 7 . The addition of NSs increased expression levels in the ER and apoplast, especially on day 3 (Fig. 2b,d). The increase in size of the ER-targeted VP6 is probably due to the additional SKDEL and 6xHis sequences present in the pTRAkc-ERH expression vector. VP6 expression was not enhanced by targeting the protein to intracellular compartments, compared to cytoplasmic accumulation (Fig. 2). These results corroborate previous studies where VP6 expression was always higher in the cytoplasm [32,33].

VP2 containing a His tag could only be detected as a $\sim 100 \mathrm{kDa}$ band at low levels in the cytoplasm at 3 and 5 dpi and when using different ODs of Agrobacterium for infiltration, and only when co-expressed with the silencing suppressor NSs (Fig. 3). Similar expression levels 


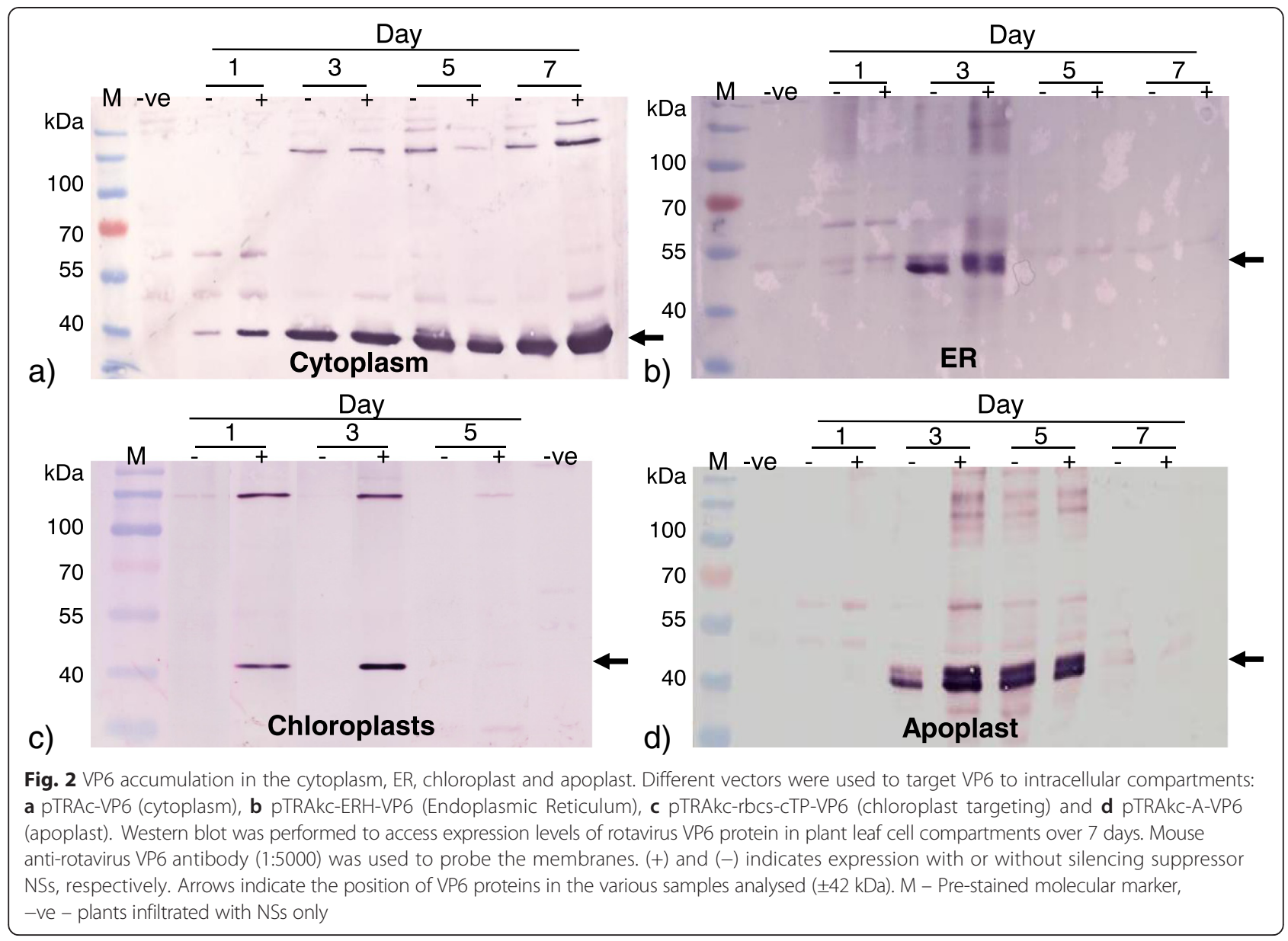

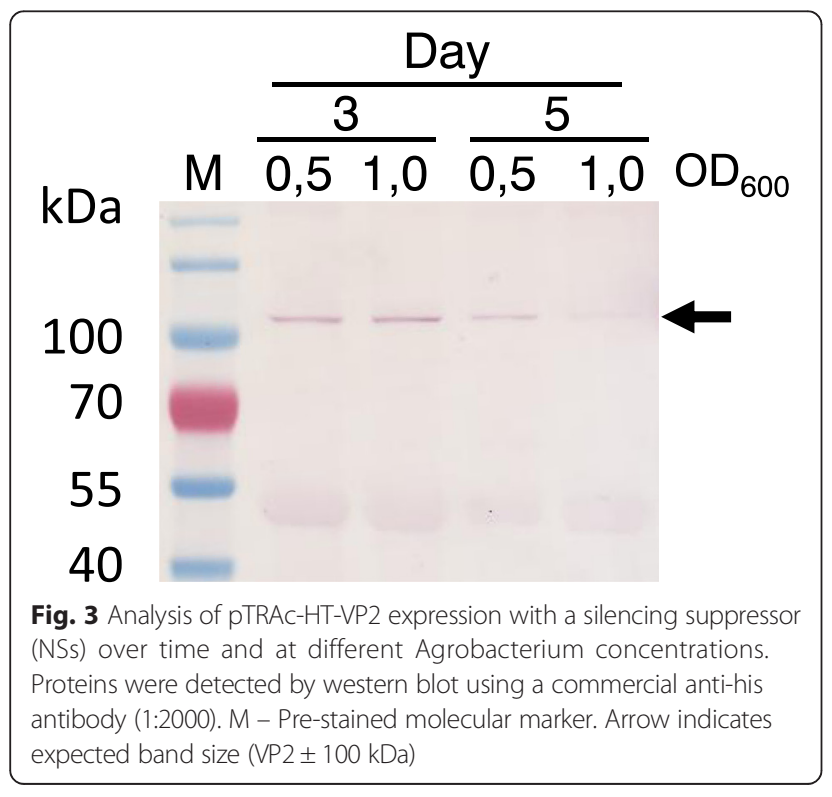

were seen for VP2 targeted to the ER, chloroplasts and apoplast (data not shown). VP2 expression without a His tag could not be explored due to lack of antibodies for detection. Low expression levels of VP2 in comparison to VP6 have been seen in both plant and insect cell expression studies [26-29].

Expression of the outer capsid proteins VP7 and VP4 was also attempted as they are essential for virion-like immunogenicity and complete particle formation. However, we could never detect expression of either protein on their own in plants, even when different pTRA expression vectors were used (data not shown). Intracellular targeting also did not have an impact on expression. In addition, plants infiltrated with VP7 exhibited yellowing symptoms from day 1 and proceeded to wilt during the time trial. Proceeding with the time trial at a lower temperature of $17{ }^{\circ} \mathrm{C}$ in order to reduce the rate of metabolic processes in the plant, and thereby hopefully lower toxicity levels of the protein, was also unsuccessful. Co-infiltrating VP7 with VP2 and VP6 at day 3 was also tried, to see if proteins could be expressed and then immediately assemble with VP2 and VP6 to form VLPs: this also failed. 
Difficulties in expressing recombinant VP7 have been previously described for E. coli and eukaryote cell expression systems, where VP7 was shown to be toxic for the cells $[37,38]$. VP7 contains a natural ER signal sequence that retains it on the ER membrane [39]. It has been suggested that, once in the ER membrane, VP7 causes an increase in the amount of $\mathrm{Ca}^{2+}$ inside the ER which leads to a disruption in cell $\mathrm{Ca}^{2+}$ homeostasis [40]. These dysregulations in $\mathrm{Ca}^{2+}$ concentrations could be responsible for the cytotoxicity and cell death responses observed when expressing VP7 [41]. Despite some studies having reported expression of VP7 in transgenic potatoes and insect cells, it is important to note that these used simian rotavirus VP7 [42] and human group A G1 VP7 [43-45], while our work focused on human rotavirus G9 VP7. Amino acid differences between the proteins in specific domains could be responsible for this effect in $N$. benthamiana. Future studies should focus on identifying these toxic domains and altering them without interfering with protein structure or immunogenicity.

Since VP4 or VP7 could not be expressed in our system, we engineered fusion proteins that would incorporate the highly immunogenic region of VP4 - located in the VP8* segment - into the structural protein VP6: the idea was that this could result in a protein that would assemble into VLPs without the glycoprotein VP7, while still presenting protective VP4 epitopes. In 2008, Istrate et al. showed that VP2 fused to VP8* successfully formed VLPs when expressed in a baculovirus system with VP6 and VP7, and that these particles induced rotavirusspecific serum IgG and IgA in mice [46].

The whole VP8* encoding sequence was inserted upstream of and in-frame with the amino-terminus of the VP6 ORF to create VP8*/6. VP8*/6 expression was confirmed by western blot using a commercial anti-VP6 antibody (1:5000) (Fig. 4a). This resulted in a band at about $75 \mathrm{kDa}$ which corresponds to the theoretical size of the fusion protein VP8*/6 (73 kDa) (Fig. 4a). We were only able to achieve VP8*/6 expression using the binary plant expression vector pEAQ-HT; previous attempts using the plasmids of the pTRA family resulted in low or non-detectable levels of the protein (data not shown). We also noted the appearance of a band of approximately $40 \mathrm{kDa}$ after day 5 (Fig. 4a and b): this corresponds exactly to the size of VP6 and was only detected using anti-VP6 antibodies. We hypothesise that, because VP8\%/6 still contains the original VP4 trypsin cleavage site, the $40 \mathrm{kDa}$ band corresponds to VP6 that is produced by cleavage of VP8*/6 by natural trypsin-like

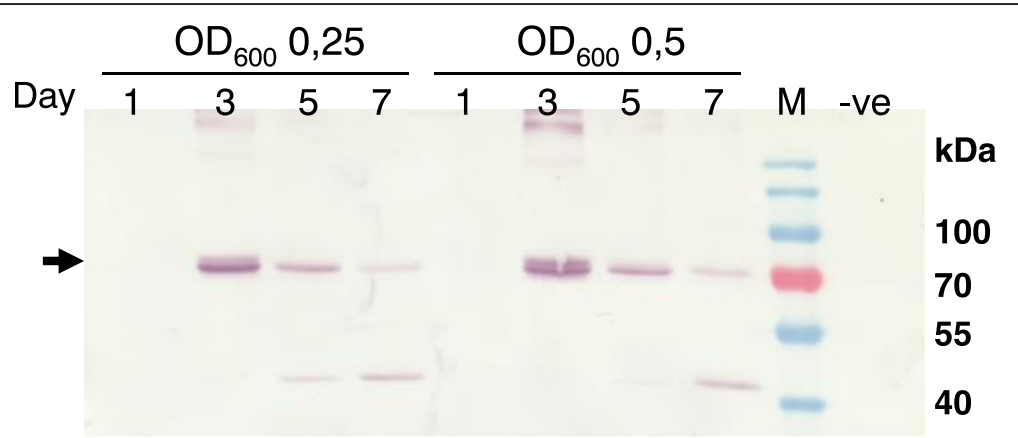

a)

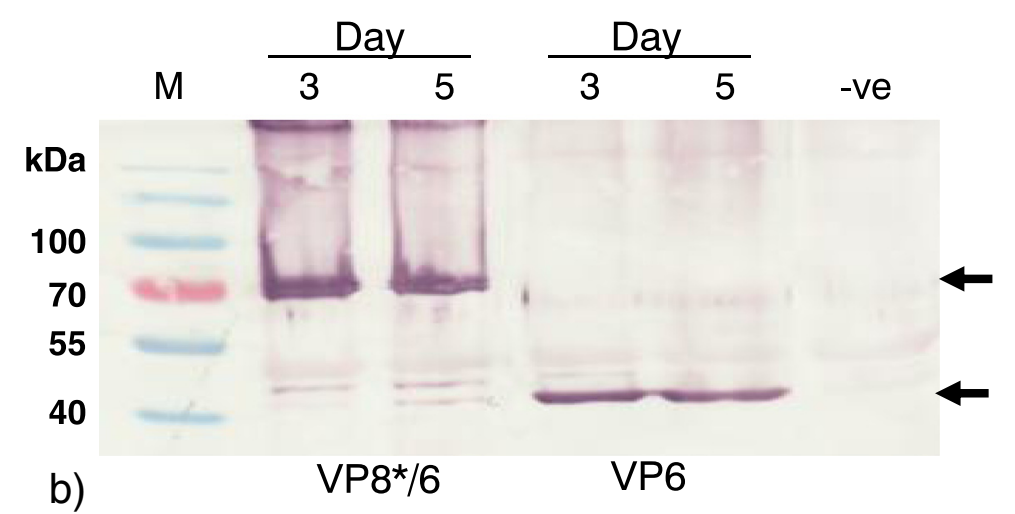

Fig. 4 Expression of the chimeric protein VP8*/6. a Western blot analysis of pEAQ-VP8*/6 expression in N. benthamiana over time at OD $600.0,25$ and 0,5 . $\mathbf{b}$ Western blot of plants infiltrated with either VP8*/6 or VP6 reveals a common band of roughly $45 \mathrm{kDa}$. Proteins were detected using commercial anti-VP6 antibody (1/5000). M - Pre-stained molecular marker, -ve - plants with infiltration media only. Arrows indicate expected band size $(\mathrm{VP} \% / 6 \pm 73 \mathrm{kDa}, \mathrm{VP} 6 \pm 42 \mathrm{kDa})$ 
proteases present in the leaves of N.benthamiana. A study using VP8" fused to VP2 also reported some VP2 degradation of VLPs containing the VP8*-2 fusion protein [47].

In order to increase chances of particle formation and to prevent protein cleavage, we designed two additional strategies for rotavirus fusion proteins. The 10 amino acid epitope situated at amino acid 1-10 of VP8* has been shown to be highly immunogenic and to elicit specific neutralizing antibodies in mice when expressed in E.coli [48]. The VP8* epitope-encoding sequence was fused by means of PCR to either the amino- or carboxyl terminus-encoding sequence of the VP6 ORF to produce $\mathrm{VP} 6 / 8^{*} \mathrm{~N}$ and $\mathrm{VP} 6 / 8^{*} \mathrm{C}$ respectively. Western blot analysis using anti-VP6 antibodies confirmed the presence of both $\mathrm{VP} 6 / 8^{*} \mathrm{~N}$ and $-\mathrm{C}$ versions in the cytoplasm of plant cells (Fig. 5a and b). Curiously, VP6/8*C appeared to be expressed more rapidly than $\mathrm{VP} 6 / 8 * \mathrm{~N}$, with highest expression levels at 3 d.p.i and 7 d.p.i., respectively. This shows that the positioning of the epitope may have an impact on protein accumulation.

Since cytoplasmic accumulation was best for both rotavirus protein expression and extraction efficiency, all co-expression work was done exclusively with proteins accumulating in the cytoplasm. VP2 is a scaffolding protein that is essential in particle formation; therefore, coexpression of VP2 with VP6, VP8\%/6, VP6/8*N and VP6/ $8 * \mathrm{C}$ was investigated. Co-expressed proteins were detected by western blot with anti-His and anti-VP6 antibodies. All proteins were detected from 3 days post infiltration (Fig. 6a and b). VP2 expression levels appeared to be higher when co-expressed with VP6 then when expressed alone: our hypothesis is that VP6 must be providing protection by assembling around the VP2 core as in natural virion morphogenesis, and preventing degradation.

To confirm VLP formation, crude extracts of infiltrated plants were fractionated by sucrose gradient centrifugation. Crude extracts were centrifuged on 10 to $60 \%$ sucrose gradients and aliquots from each fraction analysed by immunoblots probed with mouse anti-VP6 and/or mouse anti-His antibodies (Additional file 1: Figure S1). All VPs and derivatives were found in the $20-30 \%$ sucrose zone (data not shown).

\section{Electron microscopy}

Transmission electron microscopy was used to analyse particles formed by single and co-expressed recombinant proteins. VP6 alone assembled into helical and tubular structures as previously described [49, 50] (Fig. 7c). However, when co-expressed with VP2, spherical particles resembling the natural virions were seen, in both crude (Fig. 7e) and sucrose gradient-purified preparations (Additional file 2: Figure S2). The assembly of VP2/6 particles has been well documented in the insect-cell expression system [33, 51]. VP2/6 VLPs have been shown to elicit immune responses that are protective against rotavirus infections in mice [52, 53], and gnobiotic piglets [54]. Particles comprised of VP2/6/7 and 2/4/6/7 have also been expressed in insect cells; these were generally more immunogenic and prevented rotavirus infections in different animal models $[55,56]$. However, difficulty in obtaining large quantities of RV-VLPs containing the outer surface proteins VP4 and VP7 was experienced in nearly every study. Accordingly, we experimented with creating fusion proteins based on the structural properties of VP6 and the immunogenicity of VP4.

Unfortunately, despite numerous attempts, no VLPs were seen by EM for crude or purified extracts of plants expressing VP8*/6 (Fig. 7d and f), VP6/8*N or VP6/8*C (data not shown) single and co-expressed with VP2. Single expression of VP8*/6, VP6/8*N or VP6/8*C only resulted in the appearance of amorphous protein aggregates when compared with plants infiltrated with infiltration media only (negative control). We hypothesise that both the full VP8*sequence and the smaller VP8* epitope must be impeding VP6 trimerisation and particle formation. Even though few studies have been

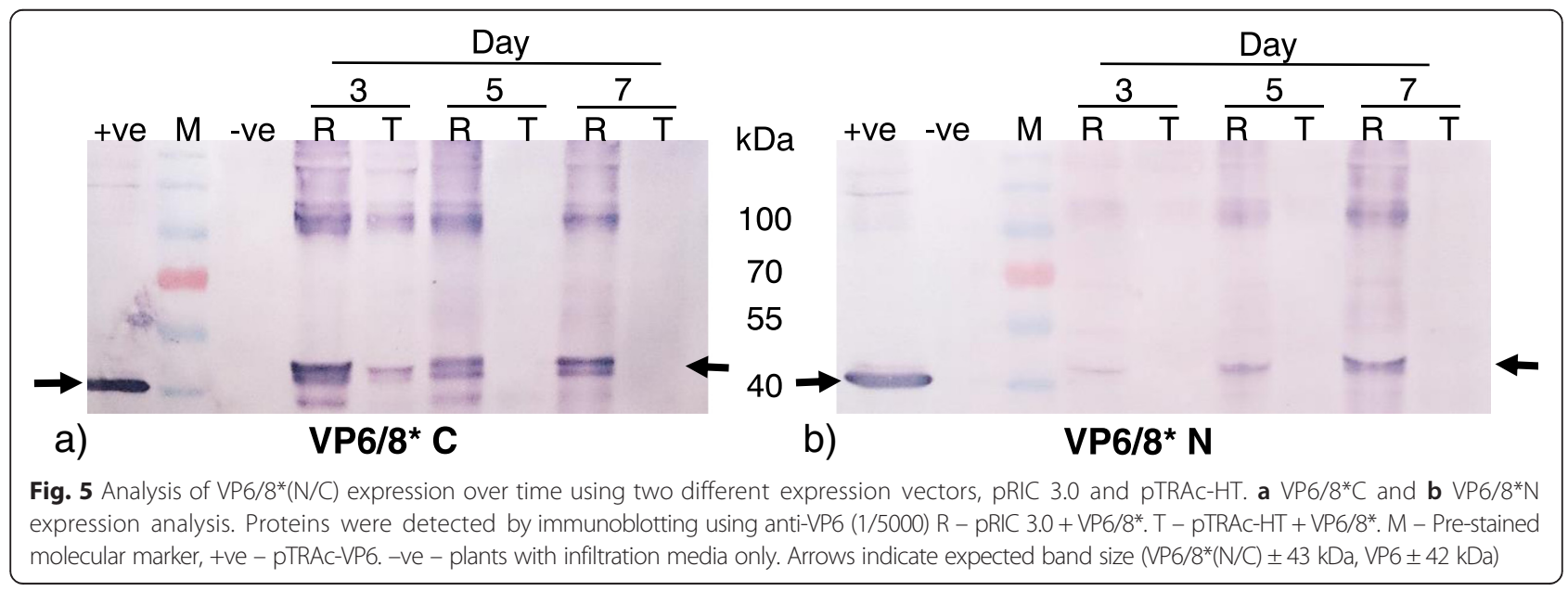




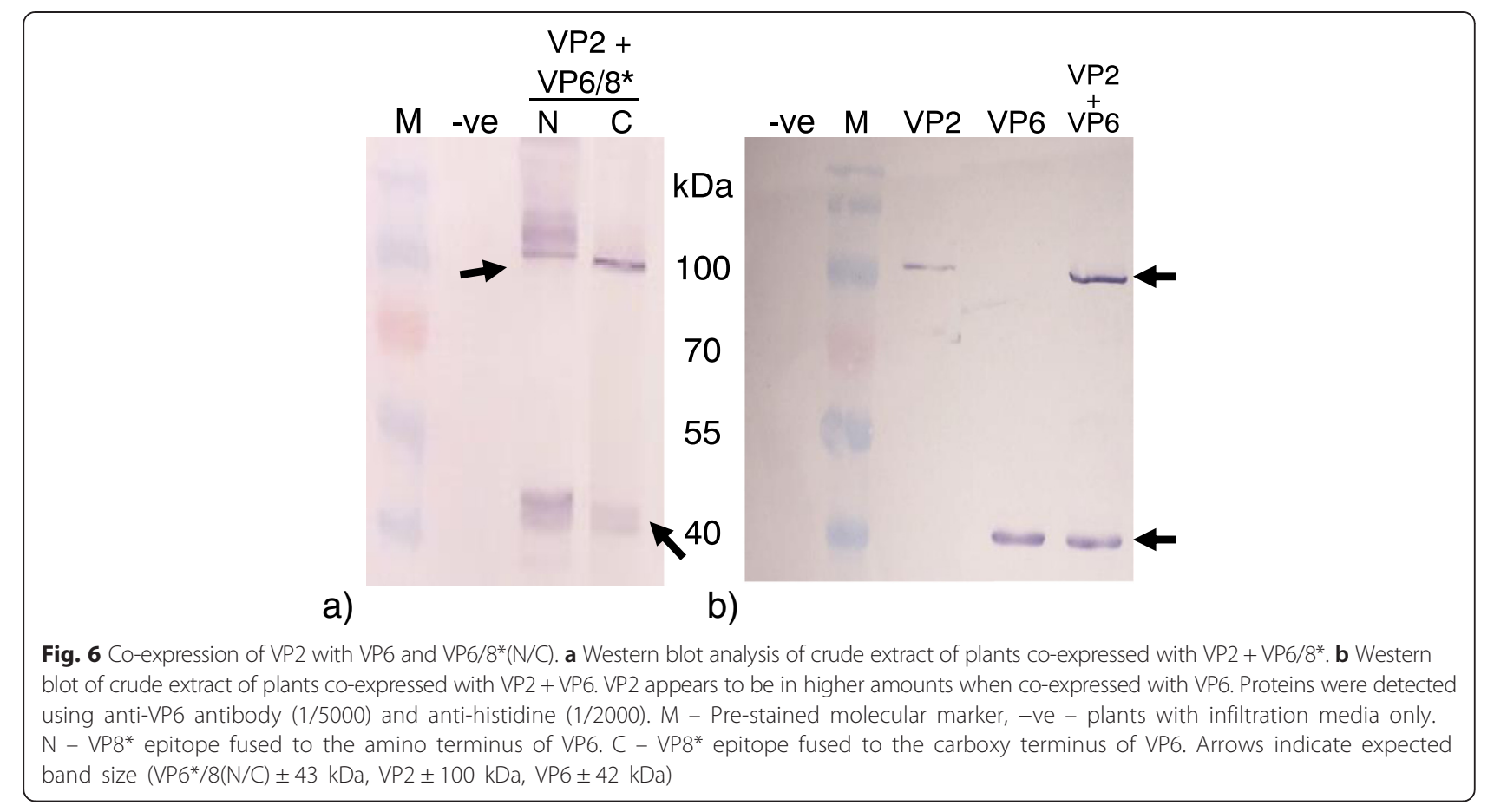

made on the structural impact of fusions with structural proteins, there have been reports of conformational changes caused by the simple addition of polyhistidine tags $[57,58]$. Moreover, a recent study on multi-epitope fusions showed that polypeptides with small differences in sequence still maintained similar levels of antigenicity, but protein folding and assembly were drastically affected [59]. Thus, the fusion proteins created in this study could still be highly immunogenic even if they do not form VLPs. Animal experiments still have to be performed to analyse the ability of these chimeric proteins as single recombinant subunit vaccines that are cheaper, safer and adapted to the South African strain G9[P6].
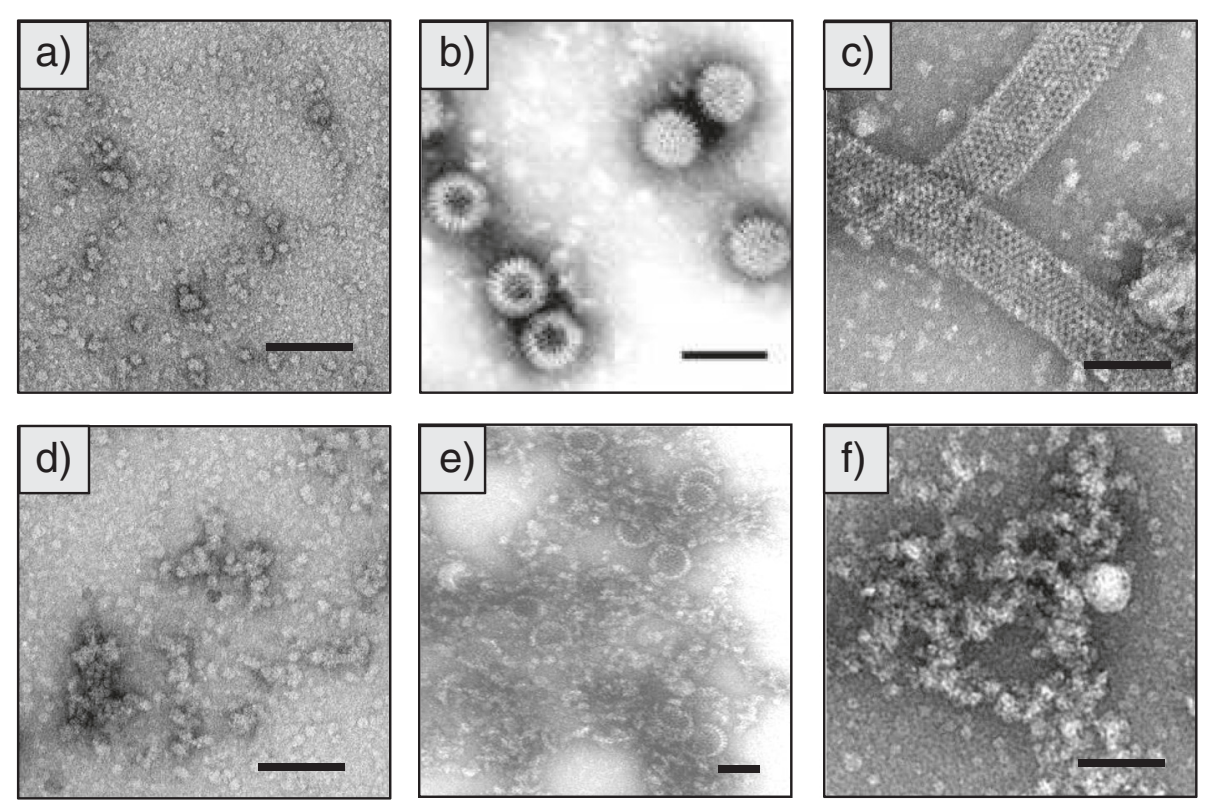

Fig. 7 Detection of rotavirus VLPs by transmission electron microscopy. a Negative control, plants infiltrated with infiltration media only. b Live rotavirus particles (Photo Credit: F.P. Williams, U.S.) (Environmental Protection Agency (EPA), 2013). c VP6. d VP8*/6. e HT-VP2 + VP6. f HT-VP2 + VP8*/6. Grids were coated with anti-VP6 (1/500). All plants were harvested at 3 dpi. Bars represent $100 \mathrm{~nm}$ 


\section{Conclusion}

Efforts to help alleviate the burden of rotavirus disease in sub-Saharan Africa and other developing countries have increased significantly in recent years. In this study, we evaluated the possibility of producing rotavirus VLPs using a plant expression system to produce a vaccine specifically adapted to the sub-Saharan African regions. We had partial success in demonstrating the capacity of the transient plant expression system to express specific rotavirus proteins. Despite the fact that no VLPs were observed for our fusion proteins, expression was detected for all chimeric proteins engineered, illustrating the versatility of plant-based systems. While this work is preliminary, we believe that it will serve as a solid basis for future studies on plant-made rotavirus vaccines for Africa.

\section{Methods}

\section{Plasmid construction}

VP2, VP4, VP6 and VP7 sequences (GenBank accession No: FJ183354, FJ183356, FJ183358 and FJ183360) from human rotavirus G9P[6] were plant codon optimised by Geneart, Germany (GenBank accession No: KT931666, KT931667, KT931668 and KT931669 respectively) [25]. The DNA was transformed in DH5- $\alpha$ chemically competent E. coli cells (E. cloni ${ }^{\mathrm{m}}$, Lucigen) as per manufacturer's instructions. DNA was extracted and genes were transformed into plant expression vectors, pTRAc (cytoplasm), pTRAkc-rbcs-cTP (chloroplast targeting), and pTRAkc-ERH (endoplasmic reticulum targeting) (Kindly supplied by R. Fischer, Fraunhofer Institute for Molecular Biology and Applied Ecology, IME, Germany) [60]. VP2, VP4 and VP6 cDNA was digested with NcoI/XhoI while VP7 was cut with AflIII/XhoI to clone into pTRAc and pTRAkc-ERH. pTRAc-HT was used for VP2, VP4 and VP7 because no specific antibodies were available; pTRAc-HT contains a 6Xhis-tag sequence upstream of the multiple cloning site (MCS), which allows for a 6Xhis-tag attached at the N-terminus of the protein of interest. For cloning in pTRAkc-ERH, a NotI restriction enzyme site was added to replace the stop codon of each the four rotavirus CDNA by PCR amplification utilising the primers as detailed in Additional file 3: Table S1. An additional vector, pTRAkc-A (apoplast), was derived from pTRAkc-ERH by cutting the vector with NcoI and XhoI thereby removing the histidine tag and the ER retention signal (SEKDEL) sequence and resulting in proteins that are targeted to the apoplast. For cloning into pTRAkc-rbcs-cTP, cDNA and vector were digested with MluI/XhoI. DNAs were sequenced to verify fidelity of the PCR reaction.

\section{Fusion protein design}

To create VP8\% $/ 6$, primers were used to introduce by PCR, a NcoI site at the $3^{\prime}$ end of the VP8* sequence located in the VP4 gene. VP6 was then cut with NcoI and ligated to VP8*; recombinant constructs were further modified by PCR to include an AgeI site on the 5' end thus allowing for cloning into the pEAQ-HT vector (Additional file 3: Table S1) [61]. For VP6/8* $\mathrm{N}$ and VP6/ $8 * \mathrm{C}$, specially designed primers were used to create the VP8*epitope on the desired terminus of the VP6 gene (Additional file 3: Table S1); each primer overlapped with 18-21 nucleotides of VP6. Forward primers added a NcoI site upstream of the VP6 whereas a XhoI site was supplemented on the $3^{\prime}$ end of the gene by the reverse primers. These sites allowed for cloning into the plant expression vectors pTRAc and pRIC 3.0. pRIC 3.0 is a self-replicating vector previously engineered and shown to increase recombinant protein expression in $N$. benthamiana [62].

Agrobacterium tumefaciens GV3101::pMP90RK was used with rotavirus pTRA-VP and pRIC-VP constructs whereas A. tumefaciens LBA4404 was used with the pEAQ-VP constructs. Electrocompetent Agrobacterium was mixed with $300 \mathrm{ng}$ of recombinant DNA in a $0.1 \mathrm{~cm}$ electrogap cuvette (BioRadTM) then electroporated in a GenePulser (BioRad) under the following settings: $1.8 \mathrm{kV}, 25 \mu \mathrm{F}$ and 200 ' $\Omega$ [63]. Incubation was allowed for $1 \mathrm{~h}$ at $27{ }^{\circ} \mathrm{C}$ in $900 \mu \mathrm{l}$ of LB before plating on LA plates containing $30 \mu \mathrm{g} / \mathrm{ml}$ kanamycin (kan), $50 \mu \mathrm{g} / \mathrm{ml}$ rifampicin (rif), $50 \mu \mathrm{g} / \mathrm{ml}$ carbenicillin (carb) was added to cultures electroporated with pTRA-VP constructs and rif and kan was added to pEAQ-VP constructs.

\section{Recombinant Agrobacterium infiltration}

Agroinfiltrations were carried out as described in Maclean et al. 2007 [60]. Briefly A. tumefeciens LBA 4404 (pEAQ-VPs) and A. tumefeciens GV3101(pMP90RK) were grown in LB with respective antibiotics and then harvested and re-suspended in induction media with antibiotics (LB, $10 \mathrm{mM}$ 2-(N-morpholino) ethanesulphonic acid MES, $2 \mathrm{mM}$ MgSO4, $20 \mu \mathrm{M}$ acetosyringone $\mathrm{pH}$ 5.6), and grown overnight at $27^{\circ} \mathrm{C}$. Overnight cultures were then spun down and resuspended in infiltration medium (10 mM MES, $10 \mathrm{mM} \mathrm{MgCl} 2,3 \%$ sucrose, $\mathrm{pH}$ 5.6, $200 \mu \mathrm{M}$ acetosyringone and sterile water) to reach the desired $\mathrm{OD}_{600}$ at $22{ }^{\circ} \mathrm{C}$ for $2-3 \mathrm{~h}$ [63]. Three week old wild type $N$. benthamiana plants were used for agroinfiltration. The VP proteins were each infiltrated in separate plants either by vacuum infiltration of whole plants or injection of recombinant Agrobacterium into the abaxial air spaces on the ventral side of plant leaves. Recombinant Agrobacterium was infiltrated either with or without the silencing suppressor LBA 4404 (pBIN-NSs). One plant was used per construct over a seven day time trial and each experiment was done in triplicate. 


\section{Protein extraction and detection}

Three leaf discs or whole leaves were harvested for each construct and ground in liquid nitrogen. Ground leaf matter was re-suspended in $1 \mathrm{x}$ sterile PBS containing Complete Protease Inhibitor (EDTA-free; Roche) at a ratio of 3:1 (buffer volume : plant weight). This was then centrifuged for $5 \mathrm{~min}$ at 25,861 $\times \mathrm{g}$ and the pellets (plant leaf matter) discarded. An additional extraction procedure was carried out on the proteins that were targeted to the apoplast utilising the pTRAkc-A vector. Whole leaves from each extraction day were vacuum infiltrated with sterile PBS containing Complete Protease Inhibitor. Individual plant leaves were suspended in PBS and put under a vacuum at $100 \mathrm{mbar}$ for $10 \mathrm{~min}$ in a vacuum tank. The leaves were then rolled and placed in spin columns (similar to Qiagen spin columns) which were placed in $2 \mathrm{ml}$ Eppendorf tubes and centrifuged at $2448 \times \mathrm{g}$ for $15 \mathrm{~min}$ and the filtrate was collected.

For western blot analysis, extracts were mixed with 5X SDS-PAGE loading buffer (2\% SDS, 0.1 M Tris$\mathrm{HCl} \mathrm{pH} \mathrm{7.5,} 20 \mathrm{mM}$ EDTA, 50 \% Glycerol, $4 \% \beta-$ mercaptoethanol, $0.02 \%$ bromophenol blue) and boiled for $2 \mathrm{~min}$ at $95{ }^{\circ} \mathrm{C}, 30 \mathrm{uL}$ of samples were loaded and separated on $12 \%$ SDS-PAGE gels and transferred onto nitrocellulose membrane by semi-dry electroblotting. VP proteins were detected using mouse anti-rotavirus VP6 antibody (US Biologicals) (1:5000), mouse antihistidine tag antibody (anti-His) (Sigma ${ }^{\circ}$ (1:2000), followed by goat anti-mouse alkaline phosphate conjugated antibodies (1:10 000 Sigma).

\section{Sucrose gradient purification of rotavirus VLPs}

Plant protein extracts were initially filtered through miracloth to remove solid plant matter. Sucrose gradients from 10 to $60 \%$ sucrose were set up in $40 \mathrm{ml}$ tubes each by creating six layers of $5 \mathrm{ml}$ of sucrose dissolved in sterile PBS ( $\mathrm{pH} 7.4)$. Clarified protein samples in 5 to $10 \mathrm{ml}$ volumes were then loaded on top of each gradient column. Ultracentrifugation at $150000 \times \mathrm{g}$ (SWTi28 swinging bucket rotor, Beckman Coulter) was carried out at $4{ }^{\circ} \mathrm{C}$ for $1 \mathrm{~h} 30 \mathrm{~min}$. At the end of the centrifugation, $2 \mathrm{ml}$ fractions were collected from the bottom of each column by tube puncture. Dot blots were then performed to determine fractions with proteins of interest. For each fraction, $1 \mu \mathrm{l}$ of sample was loaded in a grid on a nitrocellulose membrane, which was then blocked with BSA blocking buffer. Western blot analysis was then performed as usual. Proteins were probed with mouse anti-VP6 antibody (1:5000) for VP6 or anti-His for VP2 (1:2000).

\section{Electron microscopy}

To determine whether expressed proteins assembled into VLPs, transmission electron microscopy (TEM) of immuno-trapped particles was performed. Glow discharged carbon/copper grids were placed on $20 \mu \mathrm{l}$ of mouse anti-rotavirus VP6 antibody (1:500) for $5 \mathrm{~min}$ and then washed 3 times with sterile distilled water. Grids were then placed on $10 \mu \mathrm{l}$ of the protein extracts and left for $2 \mathrm{~min}$ before being washed 3 times again with sterile distilled water. Finally, grids were floated on $20 \mu \mathrm{l}$ of $2 \%$ uranyl acetate for $1 \mathrm{~min}$ before viewing under a TEM (Zeiss 912 OMEGA Energy Filter Transmission Electron Microscope, University of Cape Town).

For samples isolated from sucrose gradients, the sucrose first had to be removed by dialysis before immune-trapping on the copper grids. The sucrose fractions were placed in $10000 \mathrm{MW}$ dialysis cassettes and dialysed in sterile PBS containing $0.4 \mathrm{M} \mathrm{NaCl}$ for $4 \mathrm{~h}$ before exchanging the buffer and leaving it overnight at $4{ }^{\circ} \mathrm{C}$ with stirring.

\section{Additional files}

Additional file 1: Figure S1. Analysis of sucrose density gradient purified VP2/6. A) Western blot analysis of VP2/6 fractions using mouse anti-VP6 (1:5000) and anti-His (1:2000). B) SDS-PAGE coomassie stained gel of fractions. -ve - Fraction 16 of plants infiltrated with silencing suppressor only and sucrose gradient purified. +ve - VP6 expressed in insect cells. Arrows indicate protein band size. (PPTX $1090 \mathrm{~kb}$ )

Additional file 2: Figure S2. Transmission electron micrograph of sucrose density gradient purified VP2/6 particles. Purified VP2/6 fractions were pooled together and dialysed in high salt PBS to remove sucrose before viewing on a transmission electron microscope. Most of the VLPS remained intact but some appeared to have lost shape probably as a result of deformation due to the conditions on the EM grid. Samples were captured with mouse-anti VP6 antibody (1/500). Bar represents 100 nm. (PPTX 1531 kb)

Additional file 3: Table S1. List of primer sequences used for rotavirus chimeric protein formation. (PPTX $48 \mathrm{~kb}$ )

\section{Competing interests}

The authors declare that they have no competing interests.

\section{Authors' contributions}

FFPGP created, expressed, purified and did EM analysis of the VP8* chimeras, drafted the manuscript and revised the paper; DLRM carried out transient expression experiments of VP2, VP6, purified VLPS, did EM work and helped drafting the manuscript; AMK helped with plant expression and purification; JE created, expressed, purified and did EM analysis of the VP8* epitope chimeras; SM cloned all VP genes into plant expression vectors, AAAvD selected the viral strain, provided with sequences and helped guiding the study; EPR participated in the design of study and drafting of manuscript; $\| \mathrm{H}$ designed, coordinated and supervised the study, drafted the manuscript and revised the paper. All authors read and approved the final manuscript.

\section{Acknowledgments}

We acknowledge funding from the Department of Science and Technology (SA) - Cuba joint governmental initiative; and the Poliomyelitis Research Foundation of South Africa (PRF); Rainer Fischer (Fraunhofer IME) for the pTRA vectors, George Lomonossof (John Innes Centre) for the pEAQ vector, Marcel Prins (Laboratory of Virology, Wageningen Agricultural University) for the A. tumefaciens LBA4404 (pBIN-NSs), Mohammed Jaffer (Electron Microscope Unit, University of Cape Town) for his help with the Transmission Electron Microscope. DLRM and FFPGP were supported by awards from the PRF, AMK was supported by the National Research Foundation (NRF SA) internship system. 


\section{Author details}

${ }^{1}$ Biopharming Research Unit, Department of Molecular and Cell Biology, University of Cape Town, Rondebosch, Cape Town, South Africa.

${ }^{2}$ Biochemistry Division, North-West University, Potchefstroom, South Africa. ${ }^{3}$ Institute of Infectious Disease and Molecular Medicine, Faculty of Health Science, University of Cape Town, Cape Town, South Africa.

\section{Received: 5 August 2015 Accepted: 24 November 2015}

Published online: 02 December 2015

\section{References}

1. Bishop RF, Davidson GP. Virus-particles in epithelial-cells of duodenal mucosa from children with acute non-bacterial gastroenteritis. Lancet. 1973;2(7841):1281-3

2. Parashar UD, Hummelman EG, Bresee JS, Miller MA, Glass RI. Global illness and deaths caused by rotavirus disease in children. Emerg Infect Dis. 2003; 9(5):565-72.

3. Tate JE, Burton AH, Boschi-Pinto C, Steele AD, Duque J, Parashar UD, et al. 2008 estimate of worldwide rotavirus-associated mortality in children younger than 5 years before the introduction of universal rotavirus vaccination programmes: a systematic review and meta-analysis. Lancet Infect Dis. 2012;12(2):136-41. doi:10.1016/S1473-3099.

4. Waggie Z, Hawkridge A, Hussey GD. Review of rotavirus studies in Africa: 1976-2006. J Infect Dis. 2010;202(Supplement 1):S23-33.

5. Kosek M, Bern C, Guerrant RL. The global burden of diarrhoeal disease, as estimated from studies published between 1992 and 2000. Bull World Health Organ. 2003;81(3):197-204

6. Walker CLF, Rudan I, Liu L, Nair H, Theodoratou E, Bhutta ZA, et al. Childhood pneumonia and diarrhoea 1 global burden of childhood pneumonia and diarrhoea. Lancet. 2013;381(9875):1405-16. doi:10.1016/S0140-6736.

7. Ruiz MC, Leon T, Diaz Y, Michelangeli F. Molecular biology of rotavirus entry and replication. Sci World J. 2009;9:1476-97. doi:10.1100/tsw.2009.158.

8. Wen X, Cao D, Jones RW, Li J, Szu S, Hoshino Y. Construction and characterization of human rotavirus recombinant VP8* subunit parenteral vaccine candidates. Vaccine. 2012;30(43):6121-6. doi:10.1016/j.vaccine.2012.07.078.

9. Lentz EM, Mozgovoj MV, Bellido D, Dus Santos MJ, Wigdorovitz A, BravoAlmonacid FF. VP8* antigen produced in tobacco transplastomic plants confers protection against bovine rotavirus infection in a suckling mouse model. J Biotechnol. 2011;156(2):100-7.

10. Matthijnssens J, Otto P, Ciarlet M, Desselberger U, Van Ranst M, Johne R. VP6-sequence-based cutoff values as a criterion for rotavirus species demarcation. Arch Virol. 2012;157(6):1177-82. doi:10.1007/s00705-0121273-3.

11. Jere KC, Mlera $L, O^{\prime}$ Neill HG, Potgieter AC, Page NA, Seheri ML, et al. Whole genome analyses of African G2, G8, G9, and G12 rotavirus strains using sequence-independent amplification and 454 (R) pyrosequencing. J Med Virol. 2011;83(11):2018-42. doi:10.1002/Jmv.22207.

12. Hoshino $Y$, Kapikian AZ. Rotavirus serotypes: classification and importance in epidemiology, immunity, and vaccine development. J Health Popul Nutr. 2000:18(1):5-14

13. Matthijnssens J, Ciarlet M, McDonald SM, Attoui H, Banyai K, Brister JR, et al. Uniformity of rotavirus strain nomenclature proposed by the Rotavirus Classification Working Group (RCWG). Arch Virol. 2011;156(8):1397-413.

14. Matthijnssens J, Mino S, Papp H, Potgieter C, Novo L, Heylen E, et al. Complete molecular genome analyses of equine rotavirus A strains from different continents reveal several novel genotypes and a largely conserved genotype constellation. J Gen Virol. 2012;93(Pt 4):866-75.

15. Glass RI, Parashar UD, Bresee JS, Turcios R, Fischer TK, Widdowson MA, et al. Rotavirus vaccines: current prospects and future challenges. Lancet. 2006; 368(9532):323-32.

16. Page N, Esona M, Armah G, Nyangao J, Mwenda J, Sebunya T, et al. Emergence and characterization of serotype $G 9$ rotavirus strains from Africa. J Infect Dis. 2010;202(Suppl):S55-63.

17. Todd S, Page NA, Duncan SA, Peenze I, Cunliffe NA. Rotavirus strain types circulating in Africa: Review of studies published during 1997-2006. J Infect Dis. 2010;202(Suppl):S34-42.

18. Seheri LM, Page N, Dewar JB, Geyer A, Nemarude AL, Bos $P$, et al. Characterization and molecular epidemiology of rotavirus strains recovered in Northern Pretoria, South Africa during 2003-2006. J Infect Dis. 2010; 202(Supplement 1):S139-47.
19. Steele $A D$, Ivanoff $B$. Rotavirus strains circulating in Africa during 1996-1999: emergence of G9 strains and P[6] strains. Vaccine. 2003;21: 361-7. doi:10.1016/s0264

20. Madhi SA, Cunliffe NA, Steele D, Witte D, Kirsten M, Louw C, et al. Effect of human rotavirus vaccine on severe diarrhea in African infants. New Engl J Med. 2010;362(4):289-98. doi:10.1056/Nejmoa0904797.

21. Armah GE, Sow SO, Breiman RF, Dallas MJ, Tapia MD, Feikin DR, et al. Efficacy of pentavalent rotavirus vaccine against severe rotavirus gastroenteritis in infants in developing countries in sub-Saharan Africa: a randomised, double-blind, placebo-controlled trial. Lancet. 2010;376(9741): 606-14. doi:10.1016/s0140-6736(10)60889-6.

22. Vesikari T, Clark HF, Offit PA, Dallas MJ, DiStefano DJ, Goveia MG, et al. Effects of the potency and composition of the multivalent human-bovine (WC3) reassortant rotavirus vaccine on efficacy, safety and immunogenicity in healthy infants. Vaccine. 2006;24(22):4821-9.

23. Steele AD, Neuzil KM, Cunliffe NA, Madhi SA, Bos P, Ngwira B et al. Human rotavirus vaccine Rotarix (TM) provides protection against diverse circulating rotavirus strains in African infants: a randomized controlled trial. BMC Infect Dis. 2012;12. Doi 10.1186/1471-2334-12-213

24. Babji S, Kang G. Rotavirus vaccination in developing countries. Curr Opin Virol. 2012;2(4):443-8. doi:10.1016/j.coviro.2012.05.005

25. Potgieter AC, Page NA, Liebenberg J, Wright IM, Landt O, van Dijk AA. Improved strategies for sequence-independent amplification and sequencing of viral double-stranded RNA genomes. J Gen Virol. 2009;90(Pt 6):1423-32.

26. Hemming M, Vesikari T. Vaccine derived human-bovine double reassortant rotavirus in infants with acute gastroenteritis. Pediatr Infect Dis J. 2012;31:992.

27. El-Attar L, Oliver SL, Mackie A, Charpilienne A, Poncet D, Cohen J, et al. Comparison of the efficacy of rotavirus VLP vaccines to a live homologous rotavirus vaccine in a pig model of rotavirus disease. Vaccine. 2009;27(24): 3201-8.

28. Crawford SE, Labbe M, Cohen J, Burroughs MH, Zhou YJ, Estes MK. Characterization of virus-like particles produced by the expression of rotavirus capsid proteins in insect cells. J Virol. 1994;68(9):5945-52.

29. Bertolotti-Ciarlet A, Ciarlet M, Crawford SE, Conner ME, Estes MK Immunogenicity and protective efficacy of rotavirus 2/6-virus-like particles produced by a dual baculovirus expression vector and administered intramuscularly, intranasally, or orally to mice. Vaccine. 2003;21(25-26):3885-900.

30. Rodriguez-Limas WA, Tyo KE, Nielsen J, Ramirez OT, Palomares LA. Molecular and process design for rotavirus-like particle production in Saccharomyces cerevisiae. Microb Cell Fact. 2011;10:33.

31. Rybicki EP. Plant-made vaccines for humans and animals. Plant Biotechnol J. 2010:8(5):620-37.

32. Matsumura T, Itchoda N, Tsunemitsu H. Production of immunogenic VP6 protein of bovine group A rotavirus in transgenic potato plants. Arch Virol. 2002;147(6):1263-70.

33. O'Brien GJ, Bryant CJ, Voogd C, Greenberg HB, Gardner RC, Bellamy AR. Rotavirus VP6 expressed by PVX vectors in Nicotiana benthamiana coats PVX rods and also assembles into viruslike particles. Virology. 2000;270(2): 444-53.

34. Zhou B, Zhang Y, Wang X, Dong J, Wang B, Han C, et al. Oral administration of plant-based rotavirus VP6 induces antigen-specific lgAs, lgGs and passive protection in mice. Vaccine. 2010;28(37):6021-7.

35. Saldana S, Esquivel GF, Olivera Flores TJ, Arias N, Lopez S, Arias C, et al. Production of rotavirus-like particles in tomato (Lycopersicon esculentum L.) fruit by expression of capsid proteins VP2 and VP6 and immunological studies. Viral Immunol. 2006;19(1):42-53.

36. Takeda A, Sugiyama K, Nagano H, Mori M, Kaido M, Mise K, et al. Identification of a novel RNA silencing suppressor, NSs protein of Tomato spotted wilt virus. FEBS Lett. 2002;532(1-2):75-9.

37. Emslie KR, Miller JM, Slade MB, Dormitzer PR, Greenberg HB, Williams KL. Expression of the rotavirus SA11 protein VP7 in the simple eukaryote Dictyostelium discoideum. J Virol. 1995;69(3):1747-54.

38. McCrae MA, McCorquodale JG. Expression of a major bovine rotavirus neutralisation antigen (VP7c) in Escherichia coli. Gene. 1987;55(1):9-18.

39. Maass DR, Atkinson PH. Retention by the endoplasmic reticulum of rotavirus VP7 is controlled by three adjacent amino-terminal residues. J Virol. 1994; 68(1):366-78.

40. Zambrano JL, Diaz Y, Pena F, Vizzi E, Ruiz MC, Michelangeli F, et al. Silencing of rotavirus NSP4 or VP7 expression reduces alterations in Ca2+ homeostasis induced by infection of cultured cells. J Virol. 2008;82(12):5815-24. doi:10.1128/JVI.02719-07. 
41. Perez JF, Chemello ME, Liprandi F, Ruiz MC, Michelangeli F. Oncosis in MA104 cells is induced by rotavirus infection through an increase in intracellular Ca2+ concentration. Virology. 1998;252(1):17-27.

42. Choi NW, Estes MK, Langridge WH. Synthesis and assembly of a cholera toxin B subunit-rotavirus VP7 fusion protein in transgenic potato. Mol Biotechnol. 2005;31(3):193-202.

43. Li JT, Fei L, Mou ZR, Wei J, Tang Y, He HY, et al. Immunogenicity of a plantderived edible rotavirus subunit vaccine transformed over fifty generations. Virology. 2006;356(1-2):171-8

44. Wu YZ, Li JT, Mou ZR, Fei L, Ni B, Geng M, et al. Oral immunization with rotavirus VP7 expressed in transgenic potatoes induced high titers of mucosal neutralizing lgA. Virology. 2003;313(2):337-42.

45. Shoja Z, Tagliamonte M, Jalilvand S, Mollaei-Kandelous Y, De Stradis A, Tornesello ML, et al. Formation of self-assembled triple-layered rotavirus-like particles (tIRLPS) by constitutive co-expression of VP2, VP6, and VP7 in stably transfected high-five insect cell lines. J Med Virol. 2015;87(1):102-11. doi:10.1002/jmv.23973.

46. Istrate C, Hinkula J, Charpilienne A, Poncet D, Cohen J, Svensson L, et al. Parenteral administration of RF 8-2/6/7 rotavirus-like particles in a one-dose regimen induce protective immunity in mice. Vaccine. 2008;26(35):4594-601. doi:10.1016/j.vaccine.2008.05.089.

47. Parez N, Fourgeux C, Mohamed A, Dubuquoy C, Pillot M, Dehee A, et al. Rectal immunization with rotavirus virus-like particles induces systemic and mucosal humoral immune responses and protects mice against rotavirus infection. J Virol. 2006;80(4):1752-61. doi:10.1128/jvi.80.4.1752-1761.2006.

48. Kovacs-Nolan J, Mine Y. Tandem copies of a human rotavirus VP8 epitope can induce specific neutralizing antibodies in BALB/c mice. Biochim Biophys Acta Gen Subj. 2006;1760(12):1884-93. doi:10.1016/j.bbagen.2006.07.015.

49. Lepault J. Structural polymorphism of the major capsid protein of rotavirus. EMBO J. 2001;20(7):1498-507. doi:10.1093/emboj/20.7.1498.

50. Petitpas I, Lepault J, Vachette P, Charpilienne A, Mathieu M, Kohli E, et al. Crystallization and preliminary X-Ray analysis of rotavirus protein VP6. J Virol. 1998;72(9):7615-9.

51. Vieira HL, Estevao C, Roldao A, Peixoto CC, Sousa MF, Cruz PE, et al. Triple layered rotavirus VLP production: kinetics of vector replication, mRNA stability and recombinant protein production. J Biotechnol. 2005;120(1):72-82.

52. Saldaña S, Guadarrama FE, Flores TDJO, Arias N, López S, Arias C, et al. Production of rotavirus-like particles in tomato (Lycopersicon esculentum L.) fruit by expression of Capsid proteins VP2 and VP6 and immunological studies. Viral Immunol. 2006;19(1):42-53. doi:10.1089/vim.2006.19.42.

53. Zhou H, Guo L, Wang M, Qu J, Zhao Z, Wang J, et al. Prime immunization with rotavirus VLP 2/6 followed by boosting with an adenovirus expressing VP6 induces protective immunization against rotavirus in mice. Virol J. 2011; 8(1):3. doi:10.1186/1743-422x-8-3.

54. Yuan L, Geyer A, Hodgins DC, Fan Z, Qian Y, Chang KO, et al. Intranasal administration of 2/6-rotavirus-like particles with mutant Escherichia coli heat-labile toxin (LT-R192G) induces antibody-secreting cell responses but not protective immunity in gnotobiotic pigs. J Virol. 2000;74(19):8843-53.

55. Conner ME, Zarley CD, Hu B, Parsons S, Drabinski D, Greiner S, et al. Viruslike particles as a rotavirus subunit vaccine. J Infect Dis. 1996; 174(Supplement 1):S88-92. doi:10.1093/infdis/174.supplement_1.s88.

56. Crawford SE, Estes MK, Ciarlet M, Barone C, O'Neal CM, Cohen J, et al. Heterotypic protection and induction of a broad heterotypic neutralization response by rotavirus-like particles. J Virol. 1999;73(6):4813-22.

57. Klose J, Wendt N, Kubald S, Krause E, Fechner K, Beyermann M, et al. Hexahistidin tag position influences disulfide structure but not binding behavio of in vitro folded $\mathrm{N}$-terminal domain of rat corticotropin-releasing factor receptor type 2a. Protein Sci. 2004;13(9):2470-5. doi:10.1110/ps.04835904.

58. Chant A, Kraemer-Pecore CM, Watkin R, Kneale GG. Attachment of a histidine tag to the minimal zinc finger protein of the Aspergillus nidulans gene regulatory protein AreA causes a conformational change at the DNA-binding site. Protein Expr Purif. 2005;39(2):152-9. doi:10.1016/j.pep.2004.10.017.

59. Wang J, Lin Y, Cai P, Wang H. Effects of vector fusion peptides on the conformation and immune reactivity of epitope-shuffled, recombinant multi-epitope antigens. Protein Pept Lett. 2011;18(1):73-83.

60. Maclean J, Koekemoer M, Olivier AJ, Stewart D, Hitzeroth II, Rademacher T, et al. Optimization of human papillomavirus type 16 (HPV-16) L1 expression in plants: comparison of the suitability of different HPV-16 L1 gene variants and different cell-compartment localization. J Gen Virol. 2007;88(Pt 5):1460-9.
61. Sainsbury F, Lomonossoff GP. Extremely high-level and rapid transient protein production in plants without the use of viral replication. Plant Physiol. 2008;148(3):1212-8. doi:10.1104/pp.108.126284.

62. Regnard GL, Halley-Stott RP, Tanzer FL, Hitzeroth II, Rybicki EP. High level protein expression in plants through the use of a novel autonomously replicating geminivirus shuttle vector. Plant Biotechnol J. 2010;8(1):38-46. doi:10.1111/j.1467-7652.2009.00462.x.

63. Prins M, Resende RD, Anker C, VanSchepen A, DeHaan P, Goldbach R. Engineered RNA-mediated resistance to tomato spotted wilt virus is sequence specific. Mol Plant Microbe. 1996;9(5):416-8. doi:10.1094/Mpmi-9-0416.

\section{Submit your next manuscript to BioMed Central and we will help you at every step:}

- We accept pre-submission inquiries

- Our selector tool helps you to find the most relevant journal

- We provide round the clock customer support

- Convenient online submission

- Thorough peer review

- Inclusion in PubMed and all major indexing services

- Maximum visibility for your research 